\title{
INSECTS
}

\section{A SUMMARY OF SASKATCHEWAN BEETLES}

RONALD R. HOOPER, Box 757, Fort Qu'Appelle, SK S0G 1S0

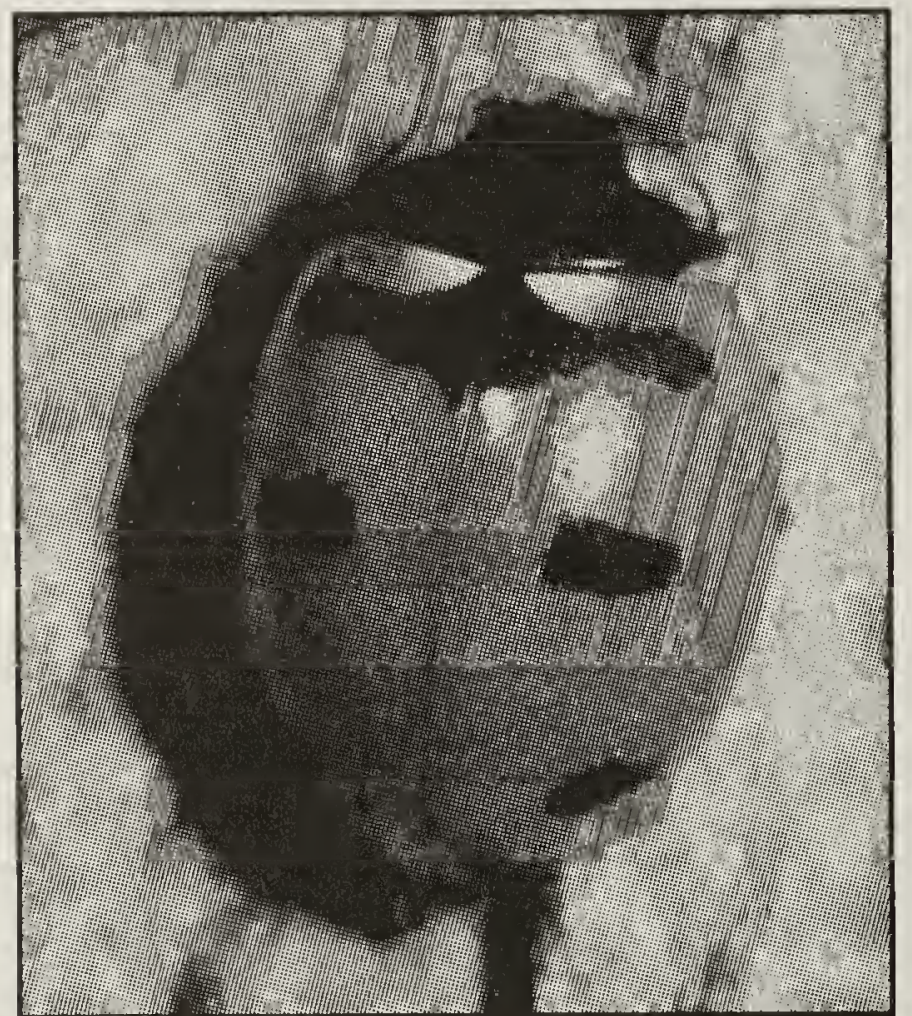

\section{Common Red Lady Beetle, Coccinella transversoguttata \\ R. Hooper}

When it comes to biodiversity, there are more species of beetles in the world than any other living creatures. Someone has aptly remarked that the Creator must have had a great fondness for beetles for He made such a variety of species. Over one third of a million species have been named worldwide, with many new ones being named every year. (As far as numbers of individuals go, however, ants would have them beat).

Beetles can be found in nearly every habitat imaginable. The more diverse habitats a beetle collector looks in, the more species he is going to find. They are on the ground, under the ground, on roots, on (or in) plant stems (or trunks), under bark, on twigs and leaves, and in tree-tops. Different kinds are in muddy water, calm water, running water, and swiftly flowing water. Special species feed on special kinds of flowering plants and on fungi. They are in nests, dung, and carrion. They are on hill-tops, in sand, and in mud. They are in barns, granaries, houses, and cellars. Our largest species of beetle is the Giant Water-scavenger Beetle (Hydrophilus triangularis). It is $39 \mathrm{~mm}$ from the front of the head to the tip of the elytra. Our smallest beetles are some of the feather-winged beetles, which are barely over one half a millimetre long.

The nucleus of the provincial beetle collection in the Royal Saskatchewan Museum (RSM) in Regina, Saskatchewan is the Cliff Shaw collection that was donated to the museum after he passed away in 1959. It contains 472 Saskatchewan species collected mainly at Yorkton, Madge Lake, and Waskesiu between 1937 and 1951. Over the years a collection of Saskatchewan beetles was built up at the Department of Agriculture Research Station in Saskatoon. By the mid-1960s this collection contained 835 species.

I started collecting beetles near Somme, $\mathrm{SK}$ in 1953. By 1969 I had 857 Saskatchewan species in the collection, and by $1980,1,161$ Saskatchewan species. These were mostly from Somme, Punnichy, and Fort Qu'Appelle. I donated my private collection to the RSM and it was incorporated into the museum's collection. The museum collection is housed in 100 glass-topped drawers in 5 cabinets. The majority of the collection has been identified by specialists in Ottawa, Edmonton, St. John's, and New York. 


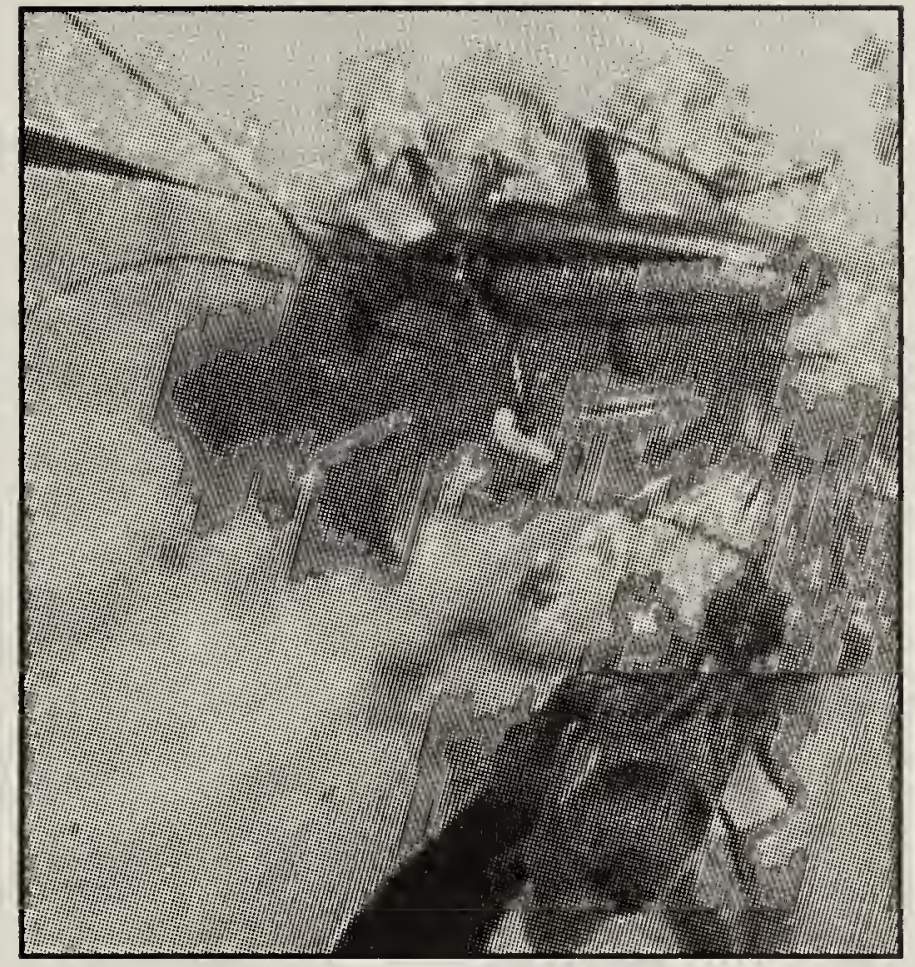

Long-horned Beetles on rose $R$. Hooper upper - Cortodera longicornis, lower - Cyrtophorus verrucosus

Beetles continued to be collected for the museum on many field trips throughout the province from 1964 on and other additional specimens are added annually. As of June of 2001 , the RSM beetle collection contained 28,316 specimens of 1,784 species. The total for the province (counting known records in other collections and unidentified species) is 2210 .

The museum has approximately 140 unidentified species in its collection. Some of these are species that have not yet been named. These wait for a specialist to revise their families or genera, and give them names. In 1995, Dr. A. Smetana published a book on the Philonthini group of rove beetles in North America. In this book, he named five new species that occur in Saskatchewan. In 1999, Dr. Y. Bousquet and S. Laplante wrote a book on the Histerid Beetles of Quebec. In it, they named two Quebec species that range west to Saskatchewan, giving us names for two more of our species.

The total number of beetle species recorded for Canada in 1991 was 7,447 species, with 7,279 in the 81 families that occur in Saskatchewan. ${ }^{1}$ The current list of 2,210 species for Saskatchewan is far from being complete; it is expected that the total for Saskatchewan could be over 5,000 species, so we have a long way to go yet!

Following is a chart of the 81 known Saskatchewan beetle families. In most cases, the numbers referred to are up to June 2001. The first column shows the total number of Saskatchewan species in each family and includes species in the RSM collection and known records in other collections. The second column gives the number of Saskatchewan species in the RSM and the third, the number of specimens in the RSM. The fourth column tells the number of species listed for Canada from each family, based on Bousquet (1991).

BOUSQUET, Y. 1991. Checklist of Beetles of Canada and Alaska. Research Branch, Agriculture Canada, Ottawa.

BOUSQUET, Y. and S. LAPLANTE. 1999. Les Coleopteres Histerides du Quebec. Association des entomologistes amateur du Quebec. Varennes, Quebec.

SHAW, C. 1953. Saskatchewan Beetles. Blue Jay 11 (1): 27.

SMETANA, A. 1995. Rove Beetles of Subtribe Philonthina of America North of Mexico. Associated Publishers, Gainsville, FL.

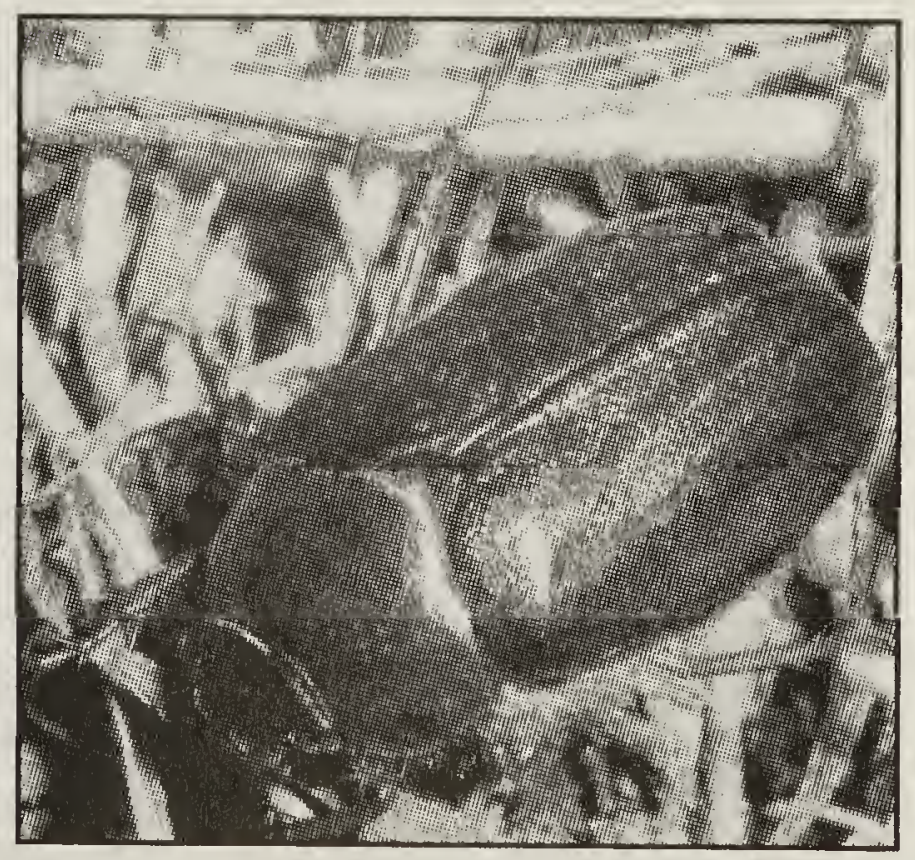

May Beetle, Phyllophaga anxia R. Hooper 
SK RSM RSM CA

Common name

Scientific name

sp. sp. Nos. sp.

1. Trachypachid Beetles

Trachypachidae

2. Ground and Tiger Beetles

Carabidae

3. Crawling Water Beetles

Haliplidae

$\begin{array}{rrrr}322 & 307 & 7,438 & 937\end{array}$

4. Predaceous Diving Beetles

Dytiscidae

11

Gyrinidae

5. Whirligig Beetles

6. Minute Moss Beetles

7. Feather-winged Beetles

8. Small Scavenger Beetles

Hydraenidae

Ptiliidae

9. Antlike Stone Beetles

10.Micropeplid Beetles

11.Carrion Beetles

12. Rove beetles

13.Shining Fungus Beetles

14. Short-winged Mold Beetles

Leiodidae

Scydmaenidae

Micropeplidae

Silphidae

Staphylinidae

Scaphidiidae

Pselaphidae

15.Water Scavenger Beetles

16. Hister Beetles

17.Plate-thigh Beetles

18.Minute Beetles

19.Marsh Beetles

20.Stag Beetles

21.Scarab Beetles

22.Pill Beetles

Hydrophilidae

Histeridae

Eucinetidae

Clambidae

Scirtidae

Lucanidae

Scarabaeidae

Byrrhidae

23.Metallic Wood-boring Beetles

Buprestidae

24. Variegated Mud-loving Beetles

25.Longtoed Water Beetles

26.Riffle Beetles

27. Click Beetles

28.Throscid Beetles

29.False Click Beetles

30.Net-winged Beetles

Heteroceridae

Dryopidae

Elmidae

Elateridae

Throscidae

Eucnemidae

Lycidae

31. Fireflies

Lampyridae

32.Soldier Beetles

Cantharidae

33. Carpet and Skin Beetles

Dermestidae

34.Bostrichid Powder-post Beetles Bostrichidae

35.Deathwatch Beetles

36.Spider Beetles

Anobiidae

Ptinidae

37. Bark-gnawing Beetles

38. Checkered Beetles

39.Soft-winged Flower Beetles

40.Dry-fungus Beetles

41.Sap Beetles

42.Root-eating Beetles

43.Flat Bark Beetles

44.Silken Fungus Beetles

45.Lizard Beetles

46.Pleasing Fungus Beetles

47. Shining Flower Beetles

Trogossitidae

Cleridae

Melyridae

Sphindidae

Nitidulidae

$\begin{array}{llll}132 & 114 & 2,842 & 262\end{array}$

$\begin{array}{llll}26 & 20 & 769 & 36\end{array}$

$\begin{array}{llll}5 & 4 & 44 & 27\end{array}$

$\begin{array}{llll}6 & 6 & 29 & 29\end{array}$

$\begin{array}{llll}38 & 15 & 129 & 123\end{array}$

$\begin{array}{llll}1 & 0 & 0 & 46\end{array}$

$\begin{array}{llll}3 & 2 & 3 & 10\end{array}$

$\begin{array}{llll}18 & 18 & 415 & 25\end{array}$

$\begin{array}{llll}243 & 187 & 1,550 & 1,084\end{array}$

$\begin{array}{llll}4 & 4 & 23 & 17\end{array}$

$\begin{array}{llll}9 & 1 & 1 & 84\end{array}$

$\begin{array}{llll}60 & 51 & 2,289 & 149\end{array}$

$\begin{array}{llll}38 & 28 & 229 & 118\end{array}$

$\begin{array}{llll}3 & 2 & 7 & 7\end{array}$

$\begin{array}{llll}2 & 2 & 4 & 7\end{array}$

$\begin{array}{llll}3 & 1 & 119 & 26\end{array}$

$\begin{array}{llll}2 & 1 & 14 & 14\end{array}$

$\begin{array}{llll}75 & 59 & 1,421 & 248\end{array}$

$\begin{array}{llll}12 & 12 & 223 & 26\end{array}$

$\begin{array}{llll}48 & 34 & 324 & 152\end{array}$

$\begin{array}{llll}14 & 3 & 656 & 25\end{array}$

$\begin{array}{llll}1 & 1 & 3 & 6\end{array}$

400,32

$\begin{array}{llll}102 & 76 & 809 & 367\end{array}$

$\begin{array}{llll}1 & 1 & 1 & 9\end{array}$

$\begin{array}{llll}4 & 3 & 3 & 32\end{array}$

$\begin{array}{llll}5 & 3 & 20 & 29\end{array}$

$\begin{array}{llll}9 & 9 & 189 & 29\end{array}$

$\begin{array}{llll}15 & 14 & 300 & 123\end{array}$

$20 \quad 18 \quad 208 \quad 46$

$\begin{array}{llll}4 & 2 & 2 & 18\end{array}$

$\begin{array}{llll}18 & 18 & 48 & 79\end{array}$

$\begin{array}{llll}8 & 3 & 28 & 16\end{array}$

$\begin{array}{llll}6 & 6 & 37 & 22\end{array}$

$\begin{array}{llll}13 & 10 & 124 & 50\end{array}$

$\begin{array}{llll}14 & 14 & 180 & 49\end{array}$

$\begin{array}{llll}37 & 34 & 264 & 101\end{array}$

$\begin{array}{lllll}\text { Rhizophagidae } & 5 & 2 & 5 & 24\end{array}$

$\begin{array}{lllll}\text { Cucujidae } & 12 & 9 & 50 & 31\end{array}$

$\begin{array}{lllll}\text { Cryptophagidae } & 24 & 27 & 157 & 66\end{array}$

$\begin{array}{lllll}\text { Languriidae } & 1 & 0 & 0 & 7\end{array}$

$\begin{array}{lllll}\text { Erotylidae } & 4 & 4 & 83 & 20\end{array}$

Phalacridae

$14 \quad 14$

123

5 
48.Cerylonid Beetles

49. Minute Fungus Beetles

50.Lady Beetles

51.Handsome Fungus Beetles

Cerylonidae

Corylophidae

$\begin{array}{llll}2 & 2 & 6 & 7\end{array}$

Coccinellidae

Endomychidae

52.Minute Brown Scavenger Beetlє Lathridiidae

53.Fruitworm Beetles

54.Hairy Fungus Beetles

55.Tetratomid Beetles

56.Minute Treef-ungus Beetles

57.False Darkling Beetles

58.Tumbling Flower Beetles

59.Wedge-shaped Beetles

60.Cylindrical Bark Beetles

61.Darkling Beetles

62.Lagriid Beetles

63.False Long-horned Beetles

64.Blister Beetles

65.False Blister Beetles

66.Borid Beetles

67.Pythid Beetles

68.Fire-colored Beetles

69.Narrow-waisted Bark Beetles

70.Antlike Flower Beetles

71.Pedilid Beetles

72.Scraptiid Beetles

73.Longhorned Beetles

74.Seed Beetles

75.Leaf Beetles

76.Fungus Weevils

77.Leaf-rolling Weevils

78.Leaf and Bud Weevils

79.Pear-shaped Weevils

80.Snout Beetles(Weevils)

81.Bark Beetles

TOTALS
Byturidae

Mycetophagidae

Tetratomidae

Ciidae

Melandryidae

Mordellidae

Rhipiphoridae

Colydiidae

Tenebrionidae

Lagriidae

Cephaloidae

Meloidae

Oedemeridae

Boridae

Pythidae

Pyrochroidae

Salpingidae

Anthicidae

Pedilidae

Scraptiidae

Cerambycidae

Bruchidae

Chrysomelidae 209

Anthribidae

Attelabidae

Rhynchitidae

Apionidae

Curculionidae

Scolytidae $\begin{array}{rrrr}3 & 2 & 9 & 11 \\ 75 & 51 & 1,343 & 162 \\ 2 & 2 & 21 & 15\end{array}$

$\begin{array}{llll}15 & 15 & 156 & 55\end{array}$

$\begin{array}{llll}1 & 1 & 1 & 1\end{array}$

$\begin{array}{llll}9 & 8 & 33 & 15\end{array}$

$\begin{array}{llll}1 & 0 & 0 & 9\end{array}$

$\begin{array}{llll}6 & 3 & 48 & 29\end{array}$

$\begin{array}{llll}12 & 12 & 22 & 55\end{array}$

$\begin{array}{llll}31 & 27 & 137 & 70\end{array}$

2000

$\begin{array}{llll}1 & 0 & 0 & 21\end{array}$

$\begin{array}{llll}30 & 27 & 490 & 135\end{array}$

$\begin{array}{llll}2 & 2 & 5 & 6\end{array}$

$\begin{array}{llll}3 & 3 & 4 & 9\end{array}$

$\begin{array}{llll}25 & 22 & 486 & 49\end{array}$

$\begin{array}{llll}3 & 3 & 11 & 13\end{array}$

$\begin{array}{llll}2 & 2 & 4 & 2\end{array}$

$\begin{array}{llll}4 & 4 & 9 & 6\end{array}$

$\begin{array}{llll}2 & 2 & 4 & 12\end{array}$

$\begin{array}{llll}1 & 1 & 2 & 10\end{array}$

$\begin{array}{llll}25 & 21 & 254 & 55\end{array}$

$\begin{array}{llll}3 & 3 & 30 & 19\end{array}$

$\begin{array}{llll}5 & 5 & 30 & 21\end{array}$

$\begin{array}{llll}77 & 71 & 776 & 354\end{array}$

$\begin{array}{llll}11 & 5 & 16 & 19\end{array}$

$\begin{array}{llll}209 & 150 & 1,948 & 569\end{array}$

$\begin{array}{llll}6 & 4 & 6 & 18\end{array}$

$\begin{array}{llll}1 & 1 & 8 & 4\end{array}$

$\begin{array}{llll}6 & 5 & 54 & 13\end{array}$

$\begin{array}{llll}18 & 11 & 27 & 35\end{array}$

$\begin{array}{llll}176 & 145 & 776 & 609\end{array}$

\begin{tabular}{rrrr}
43 & 24 & 121 & 204 \\
\hline
\end{tabular}

$\begin{array}{llll}2,210 & 1,784 & 28,316 & 7,279\end{array}$

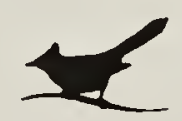

"From the sap beetle pollinators of western spicebush to the specialized scarab beetles that enter and pollinate the flowers of the giant Amazonian waterlilies, beetles are the customers and pollen vectors of choice for thousands of flowering plants on most continents....Approximately 30 families of beetles are today engaged in the pollination trade, often acting as what has been termed 'mess-and-soil pollinators.' While the label is not terribly flattering to this ancient lineage of inordinately successful insects, it does indicate their mode of entry and gustatory pursuits.

- Buchmann and Nabhan, The Forgotten Pollinators, p.95 